\section{Chromosome 22qII deletion and brain structure}

Van Amelsvoort et al (2001) report characteristic brain changes in 10 adults with velo-cardio-facial syndrome (VCFS) and 13 matched controls. The study represents an important contribution, as it is the first quantitative structural neuroimaging investigation that focuses on affected adults. The sample includes individuals with and without schizophrenia, thereby allowing generalisation to the larger population of adults with deletion $22 \mathrm{q} 11$.

In common with other investigations of subjects with neurogenetic disorders, the small sample size limits statistical power for detecting neuroanatomical differences. Null results have been interpreted and have been contrasted with a finding from our study (Eliez et al, 2000), a finding that may have been misunderstood. Specifically, the authors state that their observation of no volumetric changes in the frontal lobe among adults with VCFS is in contrast to our observation of frontal lobe abnormalities in children and that this discrepancy might indicate "delayed frontal lobe maturation which is detectable as differences in total frontal volume in childhood but subsequently normalises somewhat in adulthood ... . This interpretation implies that we found smaller frontal lobe volumes. While absolute volumes of the frontal lobe volumes were indeed smaller, adjusted frontal lobe sizes were in fact larger after statistically covarying for total brain volume, suggesting relative preservation of this structure. Preservation of frontal regions has been indicated in another recent study (Kates et al, 2001) reporting larger adjusted frontal lobe volumes and is potentially consistent with the voxel-based comparisons in the van Amelsvoort et al study, which found increased grey matter density in this region.

Two additional findings are of interest. The reported reduction in cerebellar volume is consistent with results of other recent studies (Eliez et al, 2000, 2001a), and the observed decrease in temporal lobe grey matter density among adults is in accordance with our finding of an inverse correlation between age and temporal lobe volume among affected children (Eliez et al, 2001b).

Collectively, findings from the aforementioned neuroimaging studies that rely on samples of differing age groups create an emerging picture of brain development in deletion $22 \mathrm{q} 11$ and will contribute to an increased understanding of VCFS as a genetically mediated subtype of schizophrenia.

\section{Eliez, S., Schmitt, J. E., White, C. D., et al (2000) \\ Children and adolescents with velocardiofacial \\ syndrome: a volumetric MRI study. American Journal of Psychiatry, 157, 409-4I5.}

_ , _ , _ , et al (200la) A quantitative MRI study of posterior fossa development in velocardiofacial syndrome. Biological Psychiatry, 49, 540-546.

_, Blasey, C. M., Schmitt, E. J., et al (200lb) Velocardiofacial syndrome: are structural changes in the temporal and mesial temporal regions related to schizophrenia? American Journal of Psychiatry, 158 447-453.

Kates, W. R., Burnette, C. P., Jabs, E. W., et al (200I) Regional cortical white matter reductions in velocardiofacial syndrome: a volumetric MRI analysis. Biological Psychiatry, 49, 677-684.

van Amelsvoort, T., Daly, E., Robertson, D., et al (200I) Structural brain abnormalities associated with deletion at chromosome 22qll. Quantitative neuroimaging study of adults with velo-cardiofacial syndrome. British Journal of Psychiatry, 178, 412-419.

S. Eliez, C. M. Blasey Stanford University School of Medicine, Department of Psychiatry and Behavioral Science, Stanford Psychiatry Structural Neuroimaging Laboratory, 40I Quarry Road, Psychiatry Building, Room 3360, Stanford, CA 94305-5719, USA

\section{Light therapy for seasonal affective disorder: a type II error}

Wileman et al (2001) evaluated bright white $v$. dim red light therapy for seasonal affective disorder in primary care and reported no significant difference in the proportions of responders in either group. Working on the basis of small trials having a large type II error, the group size for each group can be estimated. For most statistical tests, tables are available that show the power of the test to detect specified differences for a given $\alpha$ and sample size, as well as tables that show the required sample size to achieve selected power for specified difference and given $\alpha$ (Machin \& Campbell, 1987; Cohen, 1988). In designing a clinical trial, we should select the power $1-\beta$ to be at least 0.80 , so that there is a chance of one in five or less of missing an important difference between treatments.

The differences in response rates between the two groups even using the broad remission criterion was $16.9 \%$. To detect this difference at a significance level $\alpha=0.05$, power $80 \%, 160$ patients are required in each group (Freeman $\&$ Tyrer, 1992). However, only 57 patients in total were enrolled by Wileman et al. This reduced the power of the tests to about
$20 \%$ and strongly suggests a type II error. It would therefore be misleading to conclude that bright white light is not associated with greater improvement.

Cohen, J. (1988) Statistical Power Analysis for the Behavioral Sciences (2nd edn). Hillsdale, NJ: L. Erlbaum.

Freeman, C. \& Tyrer, P. (eds) (1992) Research Methods in Psychiatry (2nd edn), p. 52. London: Gaskell.

Machin, D. \& Campbell, M. J. (1987) Statistical Tables for the Design of Clinical Trials. Oxford: Blackwell Scientific.

Wileman, S. M. Eagles, J. M., Andrew, J. E, et a (200I) Light therapy for seasonal affective disorder in primary care. Randomised controlled trial. British Journal of Psychiatry, I78, 31I-316.

A. K. Jainer Coventry Healthcare NHS Trust, The Caludon Centre, Clifford Bridge Road, Walsgrave, Coventry CV2 2TE, UK

A. N. Singh Division of Psychopharmacology Queen's University and Kingston Psychiatric Hospital, Kingston, Ontario, Canada

N. Soni Coventry Healthcare NHS Trust Coventry, UK

\section{Pragmatic approach to the dangers of cannabis use}

I note the recent flurry of papers on the dangers of cannabis use (Ashton, 2001; Johns, 2001; MacCoun \& Reuter, 2001; Robson, 2001). As a clinician working with alcohol and drug users for 18 years, I find my mind strained by the disparity between what I read and what I see. Cannabis is almost universally used by my patients, yet only rarely can significant problems be attributed to its use. This is not to say that squirrel monkeys locked in cages with nothing to do but get stoned do not seem addicted to $\Delta^{9}$-tetrahydrocannabinol (THC) (Tanda et al, 2000; contrast with Peele, 1990). But what does that mean to humans living in complex interconnected worlds? We read that cannabis use "generally provokes relapse" in schizophrenia (Johns, 2001) but the commonest cause of relapse is schizophrenia itself. Many people choose to reduce symptoms, feel more in control or stay numb through drug use, irrespective of what we say or fear. Generally, as symptoms improve with more effective treatments, and as life's chaos subsides, alcohol, tobacco and other drug use declines or stops.

A crucial point many do not concede is that people will and do use cannabis, at an increasing frequency in most Western countries, and that dire warnings in the face of growing public acceptance will achieve 
only loss of credibility for medical bodies. Driving a car, crossing a road, moving into a new relationship, let alone taking aspirin or a tricyclic antidepressant, are all dangerous, but we balance the risk with the benefit. No doubt all the reported effects of cannabis are correctly documented. But only those who can see no benefit in cannabis seem to pounce on another piece of information to confirm what they already know to be true, that cannabis really is a deadly drug. The rest of us get along with a pragmatic, balanced view.

Ashton, C. H. (200I) Pharmacology and effects of cannabis: a brief review. British Journal of Psychiatry, 178, 101-106.

Johns, A. (200I) Psychiatric effects of cannabis. British Journal of Psychiatry, 178, 116-122.

MacCoun, R. \& Reuter, P. (200I) Evaluating alternative cannabis regimes. British journal of Psychiatry, 178, 123-128.

Peele, S. (1990) Addiction as a cultural concept. Annals of the New York Academy of Sciences, 602, 205-220.

Robson, P. (200I) Therapeutic aspects of cannabis and cannabinoids. British Journal of Psychiatry, 178, 107-115.

Tanda, G., Munzar, P. \& Goldberg, S. R. (2000) Selfadministration behavior is maintained by the psychoactive ingredient of marijuana in squirrel monkeys. Nature Neuroscience, 3, 1073-1074.

A. R. MacQueen Clinical Services Building, Bloomfield Hospital, Forest Road, Orange 2800, Australia

\section{Future of mental health services in Kosovo}

Transformation of hospital-based mental health care has been widely discussed in post-communist countries. However, there are still doubts about the efficiency of community-based care, particularly because of hospital- and drug-based traditions.

We worked between October 1999 and July 2000 as medical doctors in community shelters in Peje, Kosovo with a Czech Republic non-governmental organisation, 'People in Need'. We found that the conflict in Kosovo brought an opportunity to review the whole approach of the mental health system.

During the 1990s many Albanian physicians had been fired from jobs at public hospitals. Kosovo Albanians were often forced to seek medical care in private institutions or turn to humanitarian agencies. In May 1999, most of the Serbian minority left Kosovo, including medical staff. International institutions are now working with local doctors to fill the gap in the health care system. Kosovo, with a population of 1.8 million, had only approximately 25 psychiatrists (July 2000). Medication and hospitalisation are the only tools professionals use to deal with psychiatric disorders. The same doctors usually provide neurological and psychiatric care.

Despite this situation, the future mental health services in Kosovo will be oriented towards a community-based approach. World Health Organization adviser Liliana Urbina considers this model not only more humane but also more cost-effective and more affordable for local conditions (L. Urbina, personal communication, 2001). Community-based mental health centres are planned to organise day hospital and out-patient care, individual and group therapeutic activities, manage sheltered accommodation and a psychiatric ward for those with acute psychiatric disorders (one bed per 16 000-18 000 population). The team of 20 professionals will respond to the mental health needs of 250 000-350 000 inhabitants.

Local doctors are now being offered training in places where the concept of community-oriented psychiatry is developing such as Trieste (Italy), Asturias (Spain) and Birmingham (UK). Although local staff, who had to deal with hundreds of international organisations, often suffered from "mission fatigue" (Black \& Tosic, 1999), they seem to be attracted by this concept.

Black, M. E. \& Tosic, O. (1999) Mental health of refugees from Kosovo. Lancet, 354, 165-166.

J.Vevera, D. Pohlraich Psychiatric Clinic, Ist Medical Faculty, Charles University of Prague, Czech Republic Institute of Hematology and Blood

Transfusion, Prague, Czech Republic

\section{Suicide prevention: service contacts and coordination between primary and secondary care}

Eagles et al (2001) emphasise the problems inherent in the psychiatrist's role with regard to suicide prevention. We sympathise with their view that unrealistic expectations may lead to psychiatrists being unfairly criticised. Nevertheless, it would be a pity if we were to lose enthusiasm, without good reason, for engaging in the challenge of suicide prevention.

In quoting our paper (Vassilas \& Morgan, 1993), Eagles et al refer to our finding that younger suicides (aged $<35$ years) had a relatively low general practitioner (GP) contact rate $(20 \%)$ in the last month before they died. However, it is also important to realise that the contact rate varied greatly between subgroups of our sample. For instance, $52 \%$ of older suicides made such contact, and more specifically $68 \%$ of those over 65 years of age did so (Vassilas \& Morgan, 1994). In a later paper (Vassilas \& Morgan, 1997) we showed that $39 \%$ of all males and $76 \%$ of all females made contact with any service within the last month of their lives. This index concerned contact with either primary or secondary services rather than just with mental health services, which is the criterion used by Appleby et al (1999). Our wider index of contact presents a more encouraging, yet we believe realistic, view of our potential role in suicide prevention.

It is clear that in practice it can be difficult to coordinate primary and secondary care, but close collaboration between psychiatrist and GP, with regard to the assessment and management of suicide risk, surely warrants special attention in our attempts to become more effective in suicide prevention. The acquisition of relevant clinical skills is, as Eagles et al points out, as crucial as the epidemiological approach in this field.

Appleby, L., Shaw, J., Amos, T., et al (1999) Suicide within 12 months of contact with mental health services: national clinical survey. BMJ, 318, 1235-1239.

Eagles, J. M., Klein, S., Gray, N. M., et al (2001) Role of psychiatrists in the prediction and prevention of suicide: a perspective from north-east Scotland. British Journal of Psychiatry, I78, 494-496.

Vassilas, C. A. \& Morgan, H. G. (1993) Genera practitioners' contact with victims of suicide. BMJ, $\mathbf{3 0 7}$ 300-301.

_ \& _ (1994) Elderly suicides' contacts with their general practitioner before death. International journal of Geriatric Psychiatry, 9, 1008-1009.

_ \& _ (1997) Suicide in Avon. Life stress, alcohol misuse and use of services. British journals of Psychiatry, 170, 453-455

C.Vassilas Queen Elizabeth Psychiatric Hospital, Mindelsohn Way, Edgebaston, Birmingham BSI5 2QZ, UK

H. G. Morgan University of Bristol, Bristol, UK 